\title{
Evidence for the prolongation of aspirine induced modifications in human blood
}

\author{
R. Curini ${ }^{1}$, G. D’Ascenzo ${ }^{1}$, A. Fraioli², A. Gentili ${ }^{1}$, G. Girelli², M.A. Orrù ${ }^{1}$, and S. Materazzi ${ }^{1 *}$ \\ ${ }^{1}$ Dep. of Chemistry, University "La Sapienza”, 00185 Rome, Italy \\ ${ }^{2}$ Policlinico "Umberto I" , University "La Sapienza”, p.le A.Moro 5, 00185 Rome, Italy
}

\begin{abstract}
Aspirin is a common drug for very different purposes: it can be used for colds, for headaches, but its giving is even the usual applied therapy either for the prevention or for the pathologies of thrombosis and infarction because of the effect induced by the inhibition of thromboxane $\mathrm{A}_{2}$ synthesis. It is of relevant interest to characterize the aspirin-induced modifications in human blood to evaluate the time-dependence effects. Since the thermal analysis has been successfully applied to biological matrices to evaluate modifications and pathologies, the application of thermoanalytical techniques to the blood matrix was tempted out and the earliest results are reported: by comparing the thermoanalytical profiles of the blood samples of healthy subjects before and after aspirin giving, two different pharmacological responses can been noted and important informations about the long-term effects never reported before are proposed.
\end{abstract}

Key words. Blood - aspirin - modifications - TGA - DSC.

\section{Introduction}

Aspirine is commonly used in the case of a cold or of headache.

Blood fluidification is the usually applied therapy to thrombosis or myocardial infarction pathologies: by ticlopidine or aspirin giving, the synthesis of thromboxane $\mathrm{A}_{2}$ (platelets aggregation promoter) can be inhibited [1,2]; the antithrombotic potential of acetylsalycilic acid has been well recognised during the last 30 years because of the low incidence of thromboembolic events observed in patients under long-term treatment with this drug [3]. The cycloxygenase enzyme, that influences the platelets and the endothelial cells by means of respectively thromboxane $\mathrm{A}_{2}$ and prostacyclin can be irreversibly inhibited by the acetylsalicylic acid because it blocks the formation of $\mathrm{PGH}_{2}$, the precursor of thromboxane $\mathrm{A}_{2}$; consequently, aspirin administration inhibits the thromboxane $A_{2}$ synthesis and can so act as a platelet antiaggregating factor.

The prevention therapy of the atheromasic plaque affections is based on the same principle; while the nature and the sequence of the pathological degeneration for the atheromasic plaques is still to be clarified, instrumental techniques such as Ecodoppler of the epiaortic vessels, Transcranic Doppler, etc. have substantially modified the methodological approach to cerebrovascular pathology, as it is now possible to obtain accurate images of the dimension, composition and structure of the plaque [4]. In the blood matrix, the water interactions with the molecular systems play a foundamental role in haematic viscosity, and the inhibiting action of aspirine on thromboxane $A_{2}$ contributes to enhance the haemodynamic flow, thus lowering the risk of thromb formation or plaque breaking $[1,2,5]$.

The possibility to monitor the aspirine induced modifications in the blood is of relevant interest, and can be performed by the analysis of the water-matrix interaction energies.

Since the thermal analysis, especially thermogravimetry (TG) and differential scanning calorimetry (DSC), has been successfully applied by our group in the study of water content and water-matrix interaction energies in biological systems to evaluate modifications and pathologies [6-14], the application of the thermoanalytical techniques to the blood matrix was tempted out. The comparison among the TG and DTG curves related to healthy male subjects before and after the pharmacological treatment are reported; two different pharmacological responses have been noted and important information on long-term effects, never reported before, are proposed.

\section{Experimental section}

\section{Sample collection}

To restrict as possible biological variability, only healthy male subjects between the ages of $22 \div 28$ have been examined: as healthy subject we mean with no pathologies and having a normal range of blood values. 
The 30 healthy male subjects showed no significant difference in weight (about $70-75 \mathrm{Kg}$ ) and each analysis was performed twice to check the reproducibility.

The blood samples for each subject were collected in vacutainer tubes as follows:

- day $0=$ no pharmacological treatment (reference);

- day $7=$ after one week of aspirin administration (100 mg per day of acetylsalicylic acid);

- day 14 = one week after the end of the treatment.

Each sample was divided into two parts: one of them was centrifuged at $4000 \mathrm{rpm}$, for 5 minutes, at $4{ }^{\circ} \mathrm{C}$, to obtain the clear plasma and the corpuscular fractions; the second part was examined as whole blood.

Whole blood, plasma and corpuscular fractions were analyzed by TG and DSC techniques immediately after the sampling or the centrifuge.

\section{Instrumental}

The thermoanalytical curves were obtained by using a Perkin Elmer TGA7 thermobalance (operating range $20-1000{ }^{\circ} \mathrm{C}$ ) and a Perkin Elmer DSC7; the atmosphere was nitrogen or air, at a flow rate of $100 \mathrm{~mL} \mathrm{~min}^{-1}$; the heating rate was ranged between 5 and $40{ }^{\circ} \mathrm{C} \mathrm{min}-1$, with the best resolution at the scanning rate of $10{ }^{\circ} \mathrm{C} \mathrm{min}^{-1}$ (scan rate used in all the experiments).

\section{Results}

The thermogravimetric analysis records the weight loss of the examined sample as a function of the increasing temperature. The resulting thermal profile gives informations regarding the quantitative loss but also allows to compare the interaction energies by means of the characteristic decomposition temperature: the shift to higher temperatures is related to a stronger interaction energy.

In figure 1a the thermal profile of two whole blood samples collected at day 0 from the same subject is shown to check the possible influence of the EDTA on the blood sample (EDTA is the usual anticoagulant in the vacutainer tubes used in the clinical chemistry for the kept). The two curves represent the EDTA-added sample and the untreated blood. No differences were found due to the EDTA. The percent of water loss (first TG step in the temperature range $20 \div 200{ }^{\circ} \mathrm{C}$ ) is $79 \%$ for both samples (expanded scale, Fig. 1b). The derivative function of the TG curves (DTG curves, Fig. 1c, expanded scale) enhances the asymmetric releasing process with a convolution of many processes, one of which is more pronunced around $115^{\circ} \mathrm{C}$.

Figures $2 \mathrm{a}$ and $2 \mathrm{~b}$ report respectively the TG and the DTG curves (expanded scale $20 \div 200{ }^{\circ} \mathrm{C}$ ) of the whole blood samples of four subjects choosen as example: the water is released in many overlapped processes with a loss of $79 \%$. Because of the water evaporation, the correspond- ing DSC curves (Fig. 2c) show only one broad peak overlapping all the other processes: although the profile is similar, the four curves are shifted in temperature, as can be seen in the TG and DTG related curves.

Figure 3 shows the TG and DTG comparison among the whole blood samples obtained from the four representative subjects: the three curves in each figure represent the situation at day 0, day 7 and day 14. From these curves, exemplifing all the analyzed cases, two different behaviours can be noted:

a) (see subjects a and c) the TG and DTG curves of day 7 samples show no significant shift (or show a little temperature shift) with respect to the day 0 sample; the situation on day 14 is often the same as day 0 ;

b) (see subjects b and d) the curves of day 7 samples shift to a higher temperature (even drastically) reaching a more enhanced position on day 14 th.

Figure 4 shows the TG and DTG comparison among the plasma fraction of four subjects: the three curves in each figure represent the situation on day 0, day 7 and day 14. The water loss is $91 \%$ and the thermal behaviour can be again divided in two cases:

a) (see subjects a and c) same behaviour for the whole blood samples;

b) (see subjects b and d) the plasma fraction curves show a little shift on day 7 , while the shift to lower or to higher temperatures in the curves of day 14th is significantly marked.

Figure 5 shows the TG and DTG comparison among the corpuscular fraction of the four subjects: the three curves in each figure represent the situation on day 0, day 7 and day 14. The corpuscular fraction is the system that undergoes major modifications with again two different behaviours:

a) (see subjects a and c) no modification or little shifts in the three samples (day 0 , day 7 , day 14 );

b) (see subjects b and d) a temperature significant shift on day 7 samples with a DTG peak separation in more overlapped processes; often the pharmacological effect is still present on day 14 .

\section{Discussion}

Blood platelets can be pathologically "activated" by the release of vasoactive substances as the thromboxane $A_{2}$ that promotes the platelets aggregation [1]. After 1974, when John Vane discovered that aspirin inhibits the synthesis of prostaglandins by inactivating prostaglandin synthase, and, more specifically, the acetylsalicilate irreversibly inhibits the cyclooxygenase activity by acetylating a specific serine hydroxyl group, this drug has not only been seen as a potent anti-inflammatory agent, but it has also been widely used to prevent excessive blood clotting, which can lead to heart attacks and strokes. 


\section{Original articles}
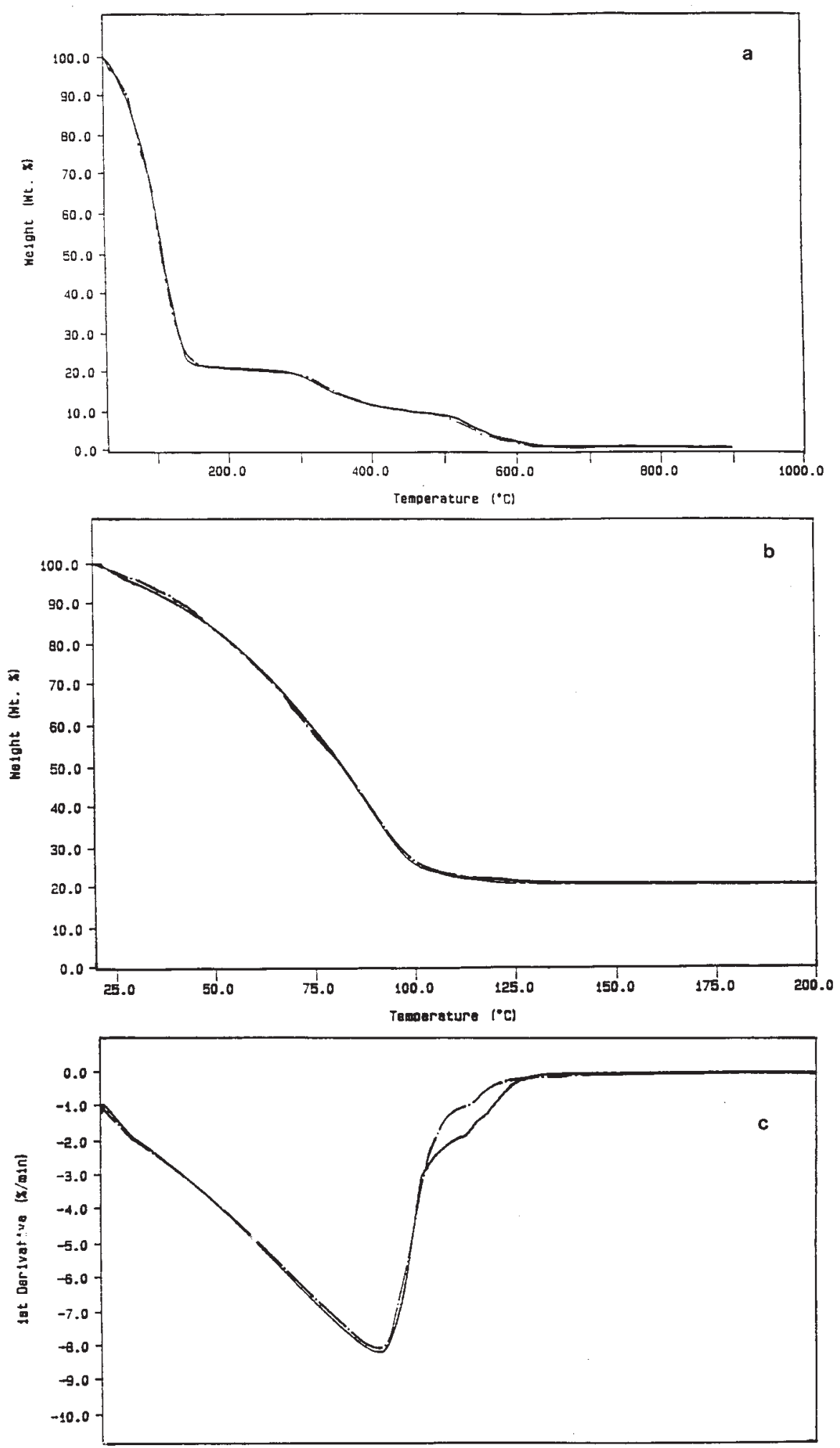

Fig. 1. Thermal profile of the whole blood samples collected at day 0: a) TG curves; b) TG curves (expanded scale); c) DTG curves. untreated blood; EDTA-added blood; Scanning rate: $10{ }^{\circ} \mathrm{C} \mathrm{min}^{-1}$; Air flow $\left(100 \mathrm{cc} \mathrm{min}{ }^{-1}\right)$. 

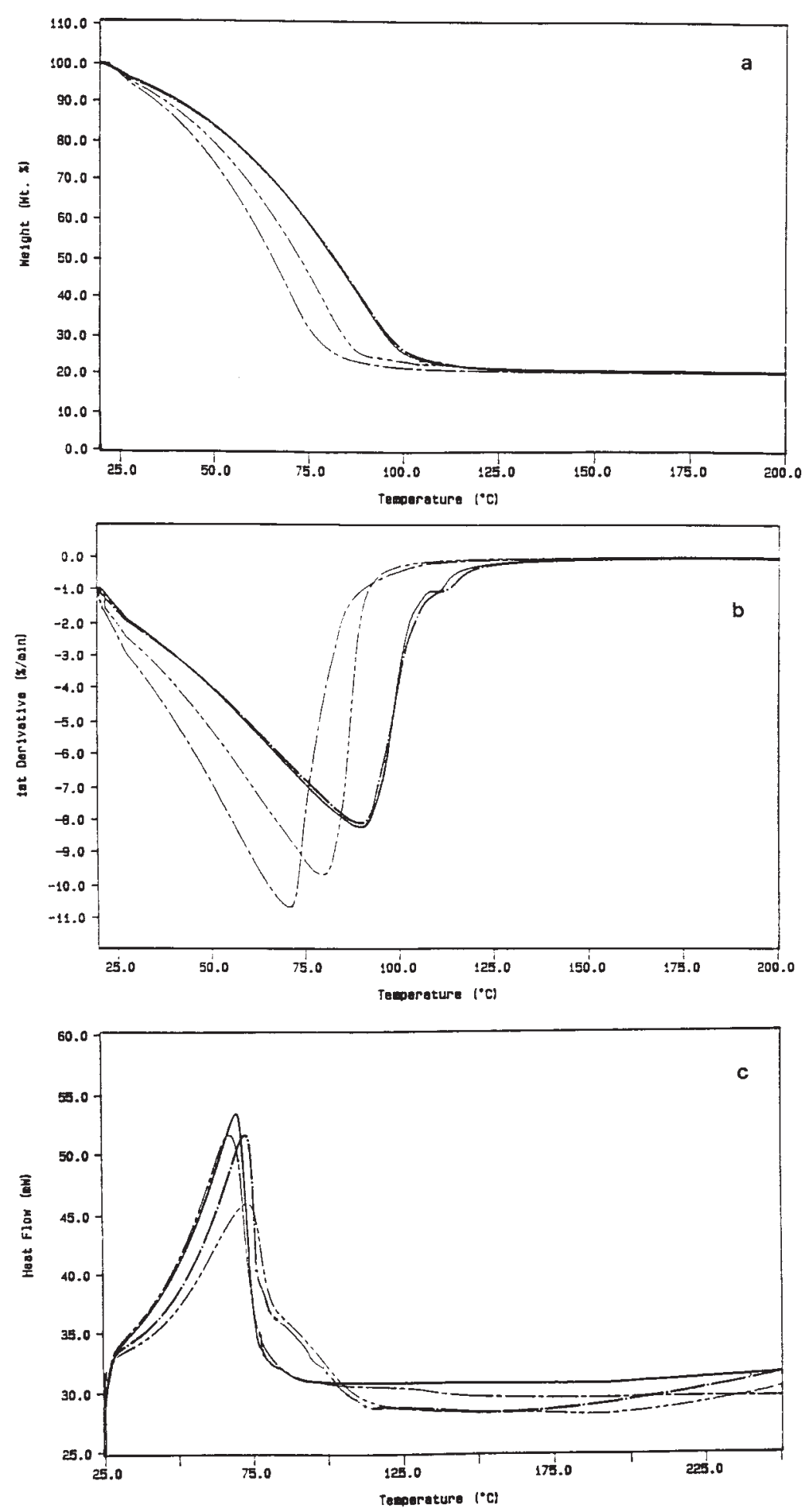

Fig. 2. Thermal profile of four subjects choosen as example (whole blood) a) TG curves; b) DTG curves; c) DSC curves. Scanning rate: $10^{\circ} \mathrm{C} \mathrm{min}^{-1}$; Air flow $\left(100 \mathrm{cc} \mathrm{min}^{-1}\right)$. 


\section{Original articles}

TG

curves

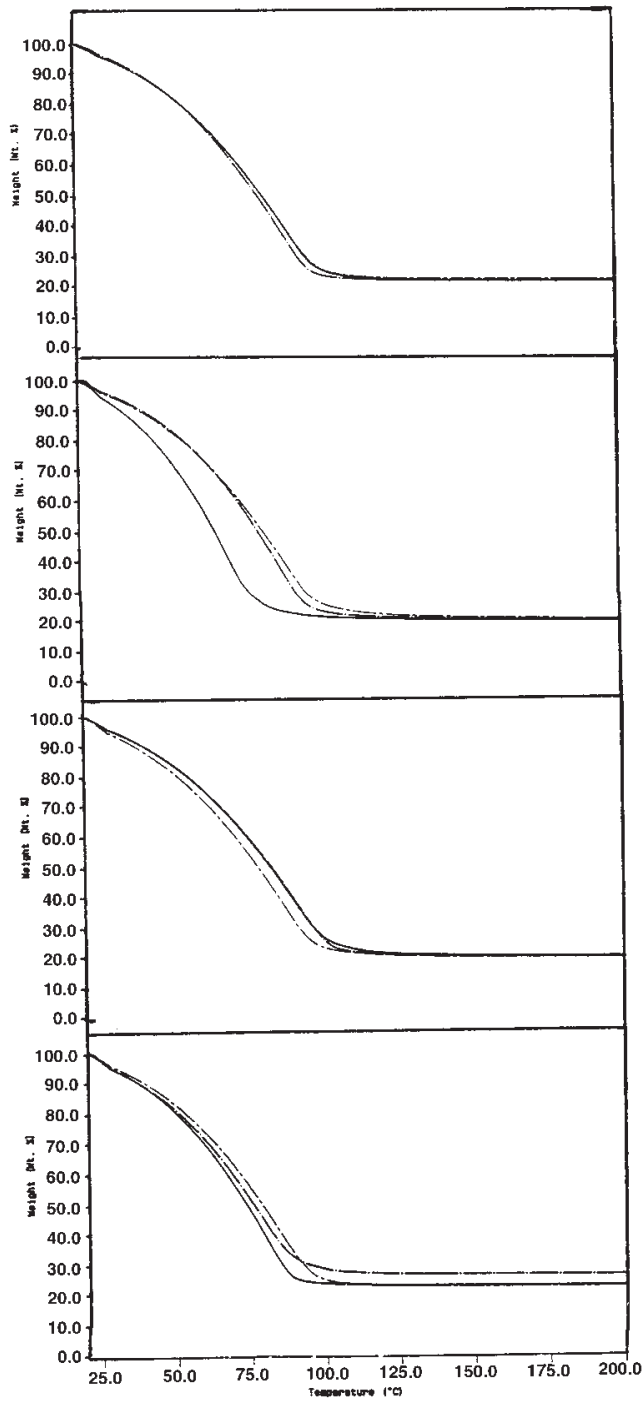

DTG

curves

a

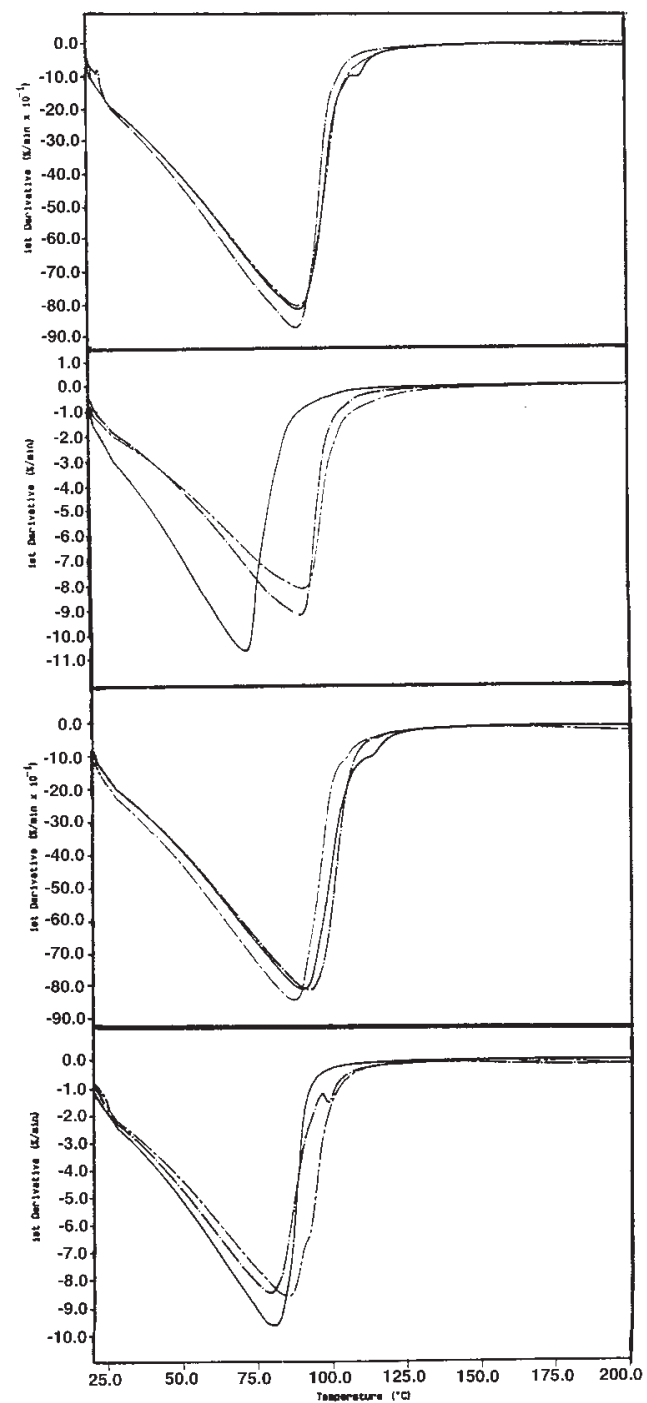

Fig. 3. Comparison among the thermoanalytical behaviour of the whole blood samples of four representative subjects: day $0 ;$ day 14; Scanning rate: $10{ }^{\circ} \mathrm{C} \mathrm{min}^{-1}$; Air flow $\left(100 \mathrm{cc} \mathrm{min}^{-1}\right)$.

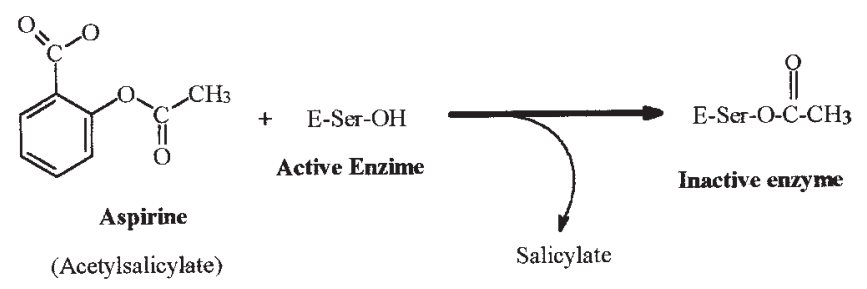

As it can be seen from the figures 4 and 5, the TG, DTG and DSC curves related to the water release (range $20 \div 200{ }^{\circ} \mathrm{C}$ ) are substantially shifted by aspirin giving in healthy subjects with a clear modification of the water-blood interactions.

In considering the factors affecting reproducibility, the thermal profiles of untreated blood samples and of EDTAadded blood samples of the same subject were compared to 
TG

curves

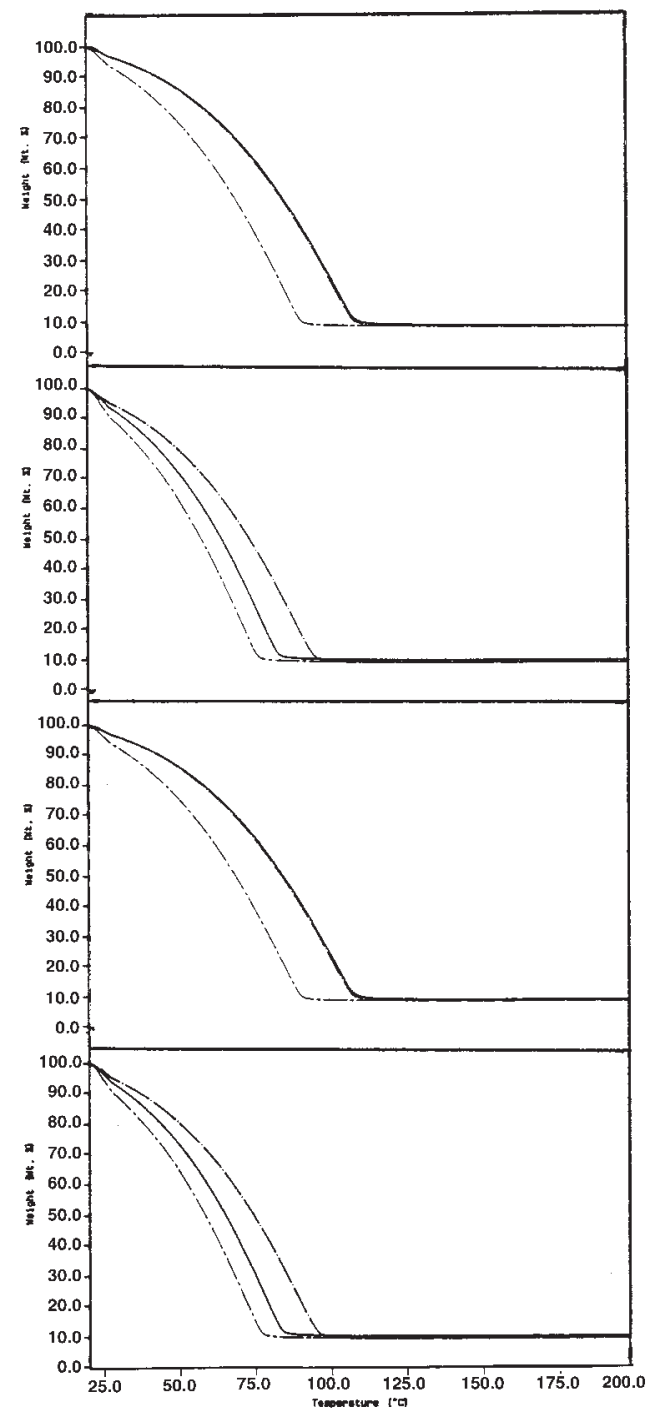

DTG curves

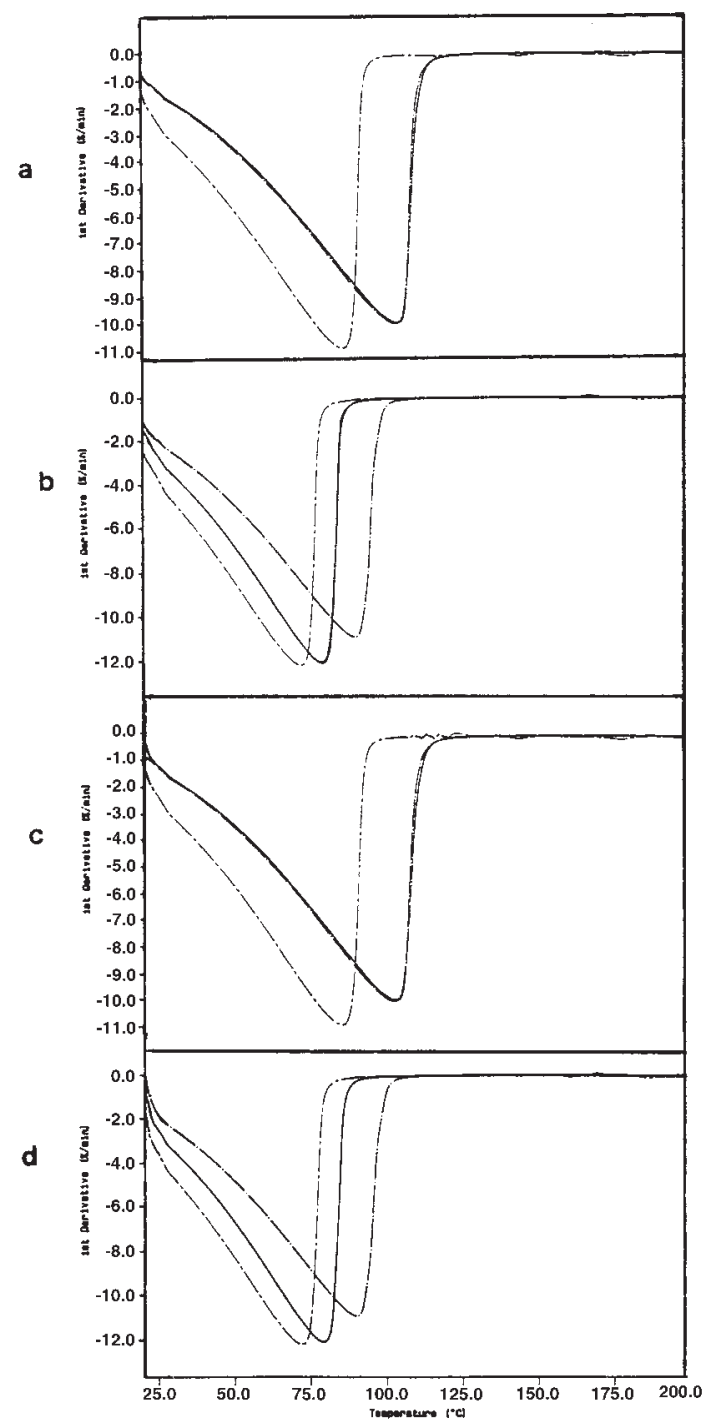

Fig. 4. Comparison among the thermoanalytical behaviour of the plasma fractions of four representative subjects: day $0 ;$ day 14. Scanning rate: $10{ }^{\circ} \mathrm{C} \mathrm{min}^{-1}$; Air flow $\left(100 \mathrm{cc} \mathrm{min}^{-1}\right)$.

check the possible influence (curves overlapped in Fig. 1). Moreover, to evaluate the possible exogenous or endogenous influence factors, with consequent modification not related to the aspirin giving, the same subjects were again analyzed about one year after the first treatment and the collected blood samples were compared with the respective time 0 samples. One representative example is reported in figure 6: the same subject gives two overlapped curves when comparing the day 0 curve with the one year later curve.
To avoid haemolysis, the blood samples were collected with a large needle syringe, and the absence of haemoglobin (signal of haemolysis) was confirmed by UV spectrometry at $405 \mathrm{~nm}$ (Soret band) or $550-575 \mathrm{~nm}$. Under these conditions, the percentage standard deviation related to the reproducibility for each subject was less than $2 \%$ in all the series. 


\section{Original articles}

TG

\section{curves}

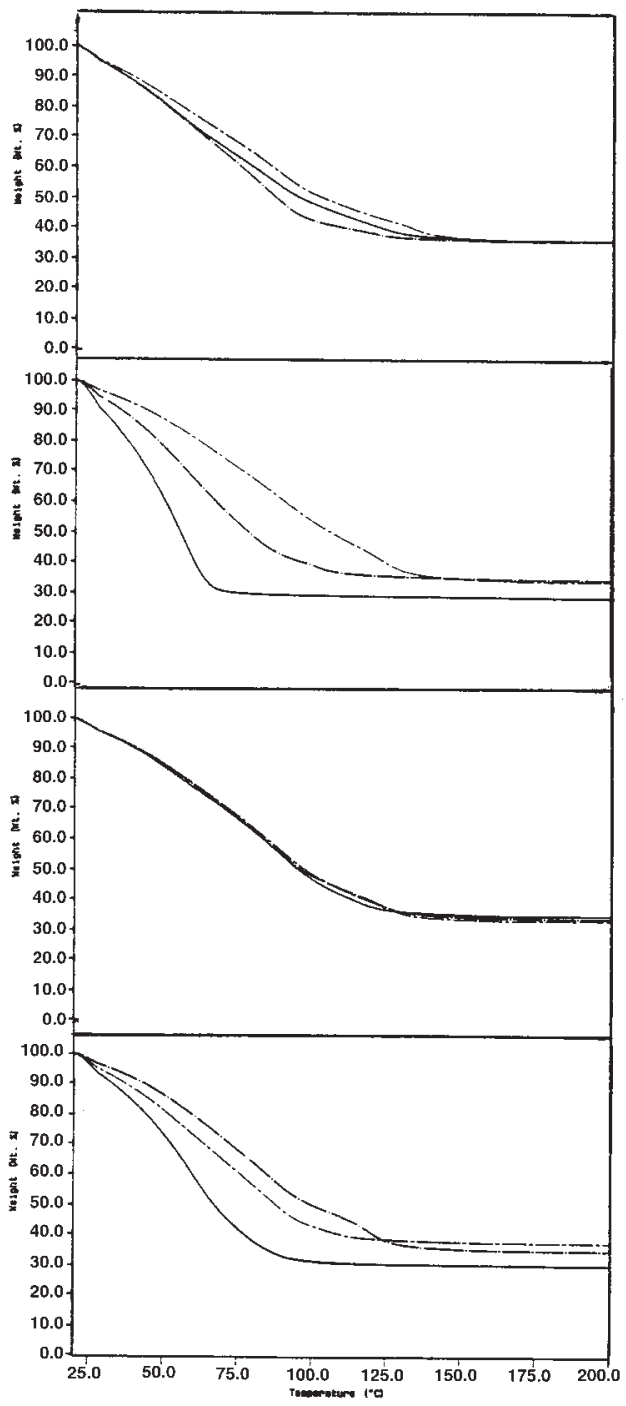

DTG

curves

a

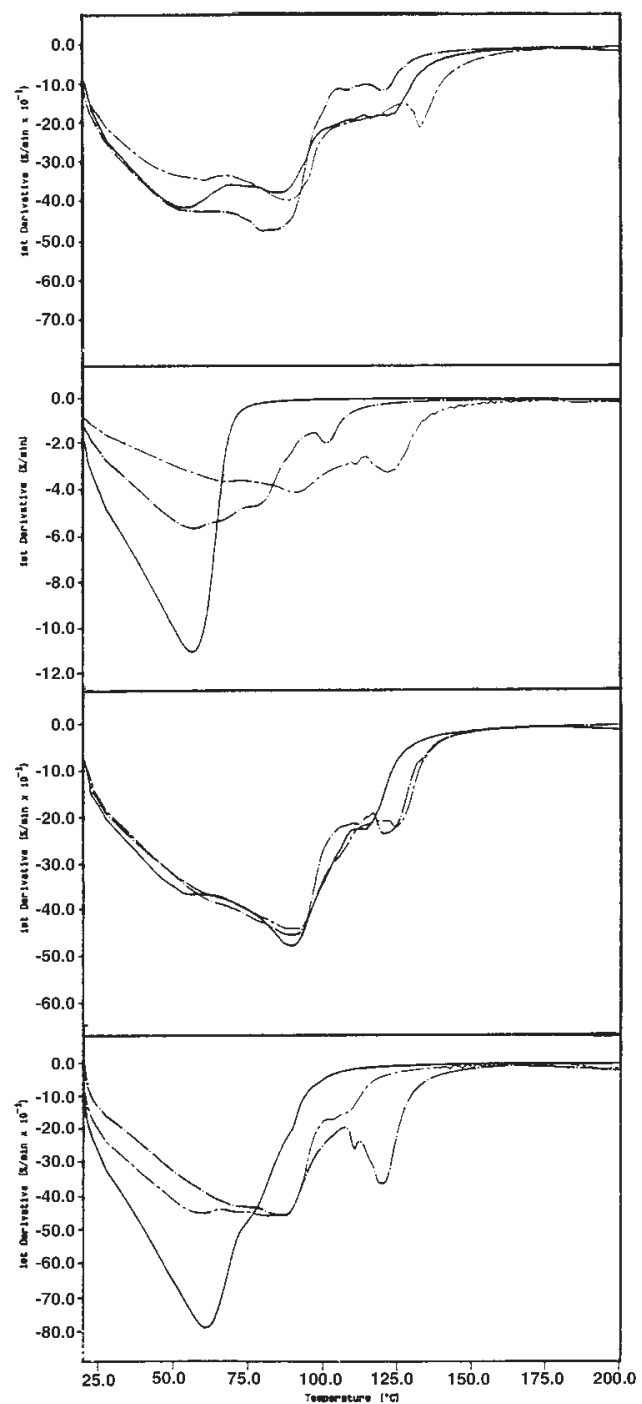

Fig. 5. Comparison among the thermoanalytical behaviour of the corpuscular fraction of four representative subjects: day 0 ; _-_-_-_-_ day 7; day 14. Scanning rate: $10{ }^{\circ} \mathrm{C} \mathrm{min}^{-1}$; Air flow $\left(100 \mathrm{cc} \mathrm{min}{ }^{-1}\right)$.

The analysis of the thermoanalytical curves enhances two different behaviours when aspirin is given:

"a" group - (see curves a and c) subjects whose curves show no significant shift among the day 0 , day 7 and day 14 collected samples;

"b" group - (see curves b and d) subjects characterized by often relevant modifications in the TG, DTG and DSC curves of day 7 and day 14 .
The signal modifications for the " $b$ " group are more marked in the corpuscular fraction, while the plasma fraction shows temperature shifts, but the percent water loss is constant; the changes in the whole blood are related to the sum of the plasma and of the corpuscular contribution.

The corpuscular fractions undergoes major modifications on day 7 and day 14, with a higher temperature shift, with separation in more overlapped processes and with a lowering of water percent loss after the aspirin giving; moreover, 


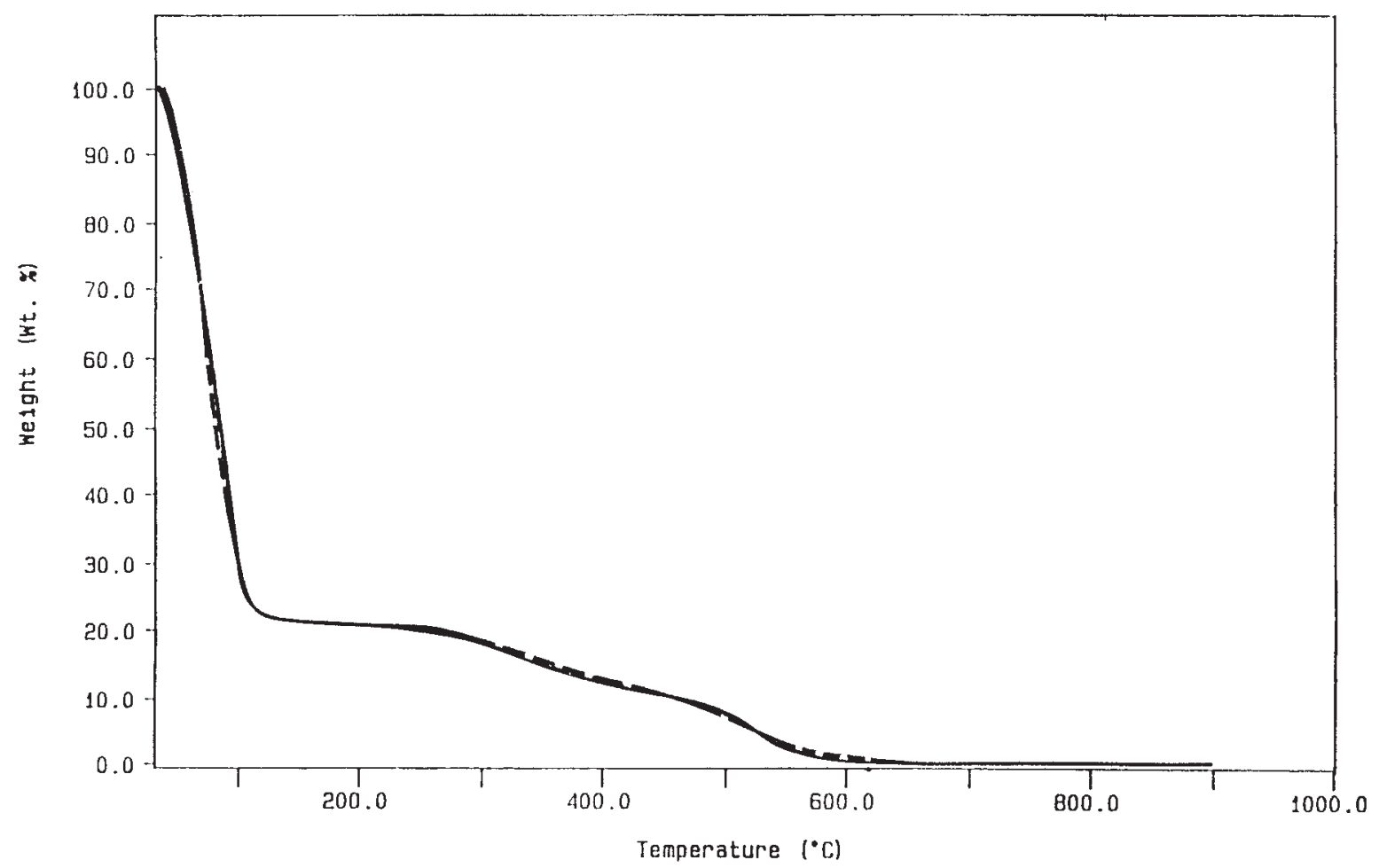

Fig. 6. Thermal profile of two blood samples from the same subject: the dotted line shows the sample kept after one year. Scanning rate: $10{ }^{\circ} \mathrm{C} \mathrm{min}-1$; Air flow $\left(100 \mathrm{cc} \mathrm{min}^{-1}\right)$.

often the day 14 curves are shifted with respect to the related day 0 curves.

These early results show that the thermoanalytical techniques are a useful tool to evaluate the aspirin induced modifications in the human blood through the modification of the water-matrix interaction energies, and suggest that in the first group of subjects the aspirin administration has a very low effect, with a consequent low modification of the interaction energies in the water-blood system; it can be assumed that the interaction between the aspirin (or its pharmacologically active methabolites) and the platelets give rise in some subjects to a new "haematic entity" that behaves as an antigen, while the aspirin or its methabolites behave as aptens; the result could be an immunitary reaction in which the aspirin or its methabolites are locked with the consequent lowering of the estimate interactions and of the pharmacological effects.

The second group of curves probably reflects the real induced modifications.

A second important aspect is related to the long-time interactions: actually, in the haemotransfusional procedure, 72 hours are assumed as the dead line of the aspirine (or similar) effects, and the blood can be collected from the donors [15]; this assumption is based on the pharmacokinetic life-time of aspirine and its methabolites (30 minutes for the aspirin and 2.5 hours for the salicylic acid and sali- cyluric acid), that would ensure a total disappearance after three days [16] .

Looking to the day 14 curves, in the subjects with enhanced modifications induced by the aspirin administration, the temperature shift is still present, with the curves related to day 0 and day 14 not overlapped, particularly in the corpuscular fraction.

These thermoanalytical data suggest that even a week is not enough to warrant the final effect of the aspirine. This phaenomenon could be explained by considering that the thromboxane $\mathrm{A}_{2}$ inhibition is irreversible, and that the regenerating capacity is estimated maximum in the $12 \%$ per day; seven days are consequently not sufficient to permit the collection of the blood samples from the donors who have taken aspirin or acetylsalicylic acid derivates.

\section{Conclusions}

These preliminary results show that the aspirin giving in healthy male subjects induces modifications that can be divided into two fundamental groups; the investigation will be completed by extending the analysis to female and to pathological subjects so to validate the thermoanalytical techniques as a useful tool in clinical investigations. 


\section{Original articles}

\section{Acknowledgements}

This research was supported by Grants (40\% and $60 \%$ ) from M.U.R.S.T. of Italy.

\section{References}

1. Ross, R. New Engl. J. Med. 1986, 314, 488.

2. Bochner, F.; Lloyd, J. Cli. Sci. 1986, 71, 625.

3. Winther, K.; Husted, S. E.; Vissinger, H. Pharmacol. Tossic. 1994, 74, 141.

4. Oster, Z. H.; Som, P. J. Nucl. Med. 1990, 31, 1055.

5. Fuster, V.; Steele, P. M.; Chesebro, J. H. J. Amer. Coll. Cardiol. 1985, 5, 175 b.

6. Curini, R.; Materazzi, S.; Menghi, G. Thermochim. Acta 1989, 153, 327.

7. Menghi, G.; Curini, R.; Materazzi, S. Cell. Molec. Biol. 1989, 35,391 .
8. Menghi, G.; Curini, R.; Materazzi, S. Cell. Molec. Biol. 1990, $36,41$.

9. Menghi, G.; Curini, R.; Materazzi, S. Cell. Molec. Biol. 1991, 37, 347.

10. Curini, R.; D’Ascenzo, G.; Bellagamba, G.; Venarucci, D. Thermochim. Acta 1991, 185, 335.

11. Curini, R.; Materazzi, S.; Marchetti, L.; Menghi, G. Cell. Molec. Biol. 1993, 39, 849.

12. Materazzi, S.; Curini, R.; Gabrielli, M. G.; Fava, L.; Menghi, G. Cell. Molec. Biol. 1994, 40, 787.

13. Materazzi, S.; Curini, R.; D’Ascenzo, G.; Magri', A. D. Thermochim. Acta 1995, 264, 75.

14. Materazzi, S.; Curini, R.; Gabrielli, M. G.; Fava, L.; Menghi G. Cell. Molec. Biol. 1996, 42, 325.

15. Mollison, P. L.; Engelfriet, C. P.; Contreras, M. In Blood Transfusion in Clinical Medicine, $10^{\text {th }}$ ed.; Blackwell Science, 1977, p. 4.

16. Mays, D. C.; Sharp, D. E.; Beach, C. A.; Kershaw, R. A.; Bianchine, J. R.; Gerber, N. J. Chromatogr. 1984, 311, 301. 